Rev. Biol. Trop. 52(1): 19-26, 2004

www.ucr.ac.cr www.ots.ac.cr www.ots.duke.edu

FORO

\title{
Hacia una lógica simple en la determinación de grupos biológicos: la especie y los grupos supraespecíficos
}

\author{
Axel P. Retana-Salazar ${ }^{1} \&$ Said A. Retana-Salazar ${ }^{2}$ \\ 1 Museo de Insectos, Universidad de Costa Rica; apretana@cariari.ucr.ac.cr \\ 2 SAx, San José, San Sebastán, Apdo. 15-1350.
}

Recibido 23-IV-2003. Corregido 20-II-2004. Aceptado 27-II-2004.

\begin{abstract}
In this paper we discuss about the utility of the species concept as real definition, particularly the Mayr concept. We propose a method for the logical separation of taxa based in the statements of the logical mathematics and the application of the sets theory to the concepts in systematic. We attempt to provide an objective methodology for the interpretation of natural groups in biology including the species as a basic group in evolution. We introduce the concept of the hypothetical ancestor as a mathematical possibility derived from the use of matrix calculations for non square matrix.
\end{abstract}

Key words: species concept, Mayr, hypothetical ancestor, non square matrix.

Con frecuencia se acusa a los biólogos de ser científicos dedicados a describir fenómenos, pero que en buena medida carecen de poder predictivo. Esto se debe en gran medida a la escasa familiaridad del biólogo con los conceptos matemáticos y físicos que permiten hacer de otras ciencias disciplinas predictivas, es decir hay una escasa familiaridad del biólogo con los conceptos del continuo tetradimensional de espacio y tiempo (Torrey 1993).

Para el biólogo tiene particular importancia la variabilidad del individuo en el tiempo, ya que un mismo individuo en sus diferentes estados ontológicos puede ubicarse en diferentes sitios en la mayoría de los sistemas (Papavero et al. 1993). Esto es sencillo de observar en insectos holometábolos donde el estado larval ocupa un sitio muy diferente al del imago. Esto va ligado a uno de los conocimientos de más antigua data y de más fácil comprobación en la biología que muestra que los individuos con todas sus características no son magnitudes constantes, sino que se transforman frecuentemente en lapsos relativamente pequeños (Hennig 1968).
Esta idea ha sido retomada recientemente y es en función de ella que se sostiene una concepción dinámica, no solamente de los procesos vitales, sino también de los organismos vivos, y de aquí se desprende una afirmación como la siguiente: "toda forma individual susceptible de ser descrita no es más que un fragmento arbitrario, determinado por el instante elegido para su consideración" (Torrey 1993).

De estas apreciaciones surge claramente que el último eslabón de los sistemas biológicos no es el individuo y con menos la especie, sino más bien el organismo tal como aparece en un instante determinado de su vida (Papavero et al. 1993). Esto es lo que se ha dado en llamar semaforonte, el cual tiene la ventaja de que al definir al semaforonte como el individuo en un lapso restringido de su existencia ayuda a comprenderlo como organismo actuante en el que se desarrollan procesos vitales (Papavero et al. 1993).

Por supuesto no existen indicadores generales de la magnitud del lapso de existencia de cada semaforonte como entidad constante y 
por lo tanto utilizable sistemáticamente, debido a que esta magnitud de tiempo es una función de la velocidad de cambio de los distintos caracteres (Papavero et al. 1993).

Sin embargo de esto se desprende que la especie será el conjunto de todos los semaforontes posibles durante su ontogenia, y en consecuencia podría enmarcarse dentro de un sistema algebraico que ayude a proponer un marco teórico de las consideraciones necesarias de la especie y de los demás taxa supraespecíficos en una forma más general y menos antojadiza que las existentes hasta el momento.

Entonces nuestra premisa básica será la siguiente, la cual se comprueba en la práctica biológica, por lo que para efectos resulta ser un axioma en este contexto teórico.

Axioma I: • Todo individuo pasa por al menos un intervalo definido de tiempo en el cual se manifiestan caracteres determinados que lo definen.

- La magnitud del o los lapsos es función de la velocidad de cambio de los caracteres, por lo que podrá haber tantos intervalos como cambios se determinen en los caracteres, siendo el mínimo posible de intervalos uno y en consecuencia la magnitud de tiempo del intervalo para el cambio de los caracteres es equivalente a la magnitud del tiempo de vida del organismo.

$\Rightarrow$ Para todo individuo existe un conjunto de variaciones espacio-temporales del individuo (I) donde cada una de esas variantes es lo que se define como semaforonte o epifenotipo (s) y el conjunto de estos epifenotipos conforma al individuo durante todo su desarrollo ontogénico siendo el menor número de estos el de 1 donde el epifenotipo corresponde a la vida entera del individuo. (según Papavero et al. 1993).

Sintaxis: los elementos morfosintáticos utilizados en la construcción lógica de este postulado son los siguientes:

$\mathrm{I}=$ individuo,

$\theta=$ conjunto de intervalos de tiempo $\theta_{\mathrm{n}}$, excluyendo $\varnothing$,
$\mathrm{F}=$ forma asociada a I en $\theta_{\mathrm{i}}$ y definida por $\mathrm{c}_{\mathrm{i}}$, $\mathrm{C}^{\prime}=$ conjunto de caracteres que definen algún objeto o estado del mismo, manteniendo la unidad y organización de la información, $\mathrm{O}=$ conjunto de poblaciones en los intervalos $\theta_{\mathrm{n}}$.

Entonces

i. $\forall \mathrm{I} \exists \theta_{\mathrm{I}}$ que es el conjunto de intervalos de tiempo asociados a $\mathrm{I} \Rightarrow \exists \mathrm{F}_{\mathrm{I}}$ determinado como la forma de I asociada a $\theta_{\mathrm{I}}$ que formará el par ordenado $\left(\mathrm{F}_{\mathrm{i}}, \theta_{\mathrm{Ii}}\right)$ que define a cada epifenotipo de I en cada tiempo i como $\mathrm{S}_{\mathrm{Ii}}=\left(\mathrm{F}_{\mathrm{i}}, \theta_{\mathrm{Ii}}\right),(1 \leq \mathrm{i} \leq \mathrm{n}, \mathrm{n} \geq 1)$ (según Papavero et al. 1993).

ii. $\forall \mathrm{O}^{\prime}=\left[\mathrm{O}^{\prime}-\varnothing\right] \Lambda \mathrm{O}^{\prime}=\left\langle\mathrm{o}_{1}, \ldots, \mathrm{o}_{\mathrm{n}}\right\rangle$, donde $\mathrm{o}_{1}$ describe a una población en un momento determinado, siendo que

$$
\bigcap_{\mathrm{i}=1}^{\mathrm{n}} \mathrm{o}_{\mathrm{n}}=\varnothing,(\mathrm{n} \geq 1)
$$

iii. De acuerdo con esto entonces todo $o_{x}=<I-$ ${ }_{x i}, \ldots, I_{x n}>,(n \geq 2)$, que serán los estados de los individuos en un momento dado de la población, entonces $\exists \mathrm{C}^{\prime}$ tal que $\mathrm{C}^{\prime}=\left[\mathrm{C}^{\prime}\right.$ $\varnothing]$ definido como el conjunto de los caracteres que definen a $\mathrm{O}^{\prime} \mathrm{y}$ donde $\mathrm{C}^{\prime}=$ $\left\langle\mathrm{c}_{1}, \ldots \mathrm{c}_{\mathrm{n}}\right\rangle$, para un tal $\mathrm{O}^{\prime}=\left\langle\mathrm{o}_{1}, \ldots, \mathrm{o}_{\mathrm{n}}\right\rangle$, $(n \geq 1)$.

iv. Si los corolarios anteriores son verdaderos entonces

$$
\forall \mathrm{o}_{\mathrm{x}} \exists \mathrm{c}_{\mathrm{ox}}
$$

que define los caracteres de la población.

v. $\forall \mathrm{o}_{\mathrm{x}} \Lambda \mathrm{c}_{\mathrm{ox}} \exists \theta_{\mathrm{ox}}$ de tal forma que se puede establecer la siguiente relación de orden $\left(\mathrm{o}_{\mathrm{x}}, \mathrm{c}_{\mathrm{ox}}, \theta_{\mathrm{ox}}\right)=$ a una población separada de otras por sus características definidas para ella en un tiempo propio asociado a ella y a esas características, en consecuencia podemos definir al conjunto de relaciones de orden entre estas tres variables expresado como 
$\mathrm{e}=\left(\mathrm{o}_{\mathrm{i}}, \mathrm{c}_{\mathrm{oi}}, \theta_{\mathrm{oi}}\right),(\mathrm{i}=1 \ldots \mathrm{n}, \mathrm{n} \geq 1)$, entendiéndose con esto que el conjunto e es equivalente a la unidad independiente de evolución en un tiempo dado, con unas características dadas y un número de individuos determinado y finito, que sería lo que podemos llamar especie.

vi. $\forall \mathrm{e}_{1}, \mathrm{e}_{2} \exists \mathrm{c}_{\mathrm{e} 1}, \mathrm{c}_{\mathrm{e} 2} \Leftrightarrow \mathrm{c}_{\mathrm{e} 1}-\mathrm{c}_{\mathrm{e} 2} \neq \varnothing \Leftrightarrow \mathrm{e}_{1} \neq \mathrm{e}_{2}$. Con lo que se establece en forma clara definitiva la existencia de una correspondencia de caracteres excluyentes que separa las especies, es decir que cada especie tendrá al menos un carácter o una combinación de ellos que hace que el conjunto c asociado a ella sea único y diferente de exhibido por cualquier otra unidad evolutiva llamada especie.

vii. $\mathrm{E}=\left\langle\mathrm{e}_{1}, \ldots, \mathrm{e}_{\mathrm{n}}>(\mathrm{n} \geq 1)\right.$

será un E-linaje donde todos sus elementos estarán subordinados por la relación cinclusión y resulta en un inf-reticulado, o conjunto acotado inferiormente que es una cadena donde $\mathrm{c}_{\mathrm{en}} \leq \mathrm{c}_{\mathrm{en+1}},(\mathrm{n} \geq 2)$ (según Papavero et al. 1993), donde si $\mathrm{c}_{\mathrm{en}}$ es el conjunto de caracteres que describen a $e_{n} y$ este es el origen será conocido como ancestro y su relación con los descendientes descrita como a, y se representa como $e_{n} \mathbf{a}$ $\mathrm{e}_{\mathrm{n}+1} \mathrm{y}$ será denominado como $\mathrm{e}_{\mathrm{anc}}$.

viii. Si además existe un conjunto acotado inferiormente descrito como un inf-reticulado donde $n \geq 3$ definido por la relación de orden c-inclusión que no resulta en una cadena pero está formado por varias cadenas que son conjuntos acotados inferiormente relacionados entre sí y determinados por un origen común, y si se puede determinar o inferir la relación a entre uno o varios de los constituyentes hablamos de un E-genos y en ambos casos se cumple que

$$
\bigcap_{\mathrm{i}=1}^{\mathrm{n}} \mathrm{c}_{\mathrm{n}} \neq \varnothing
$$

La utópica definición de especie: Una pregunta que debemos plantearnos antes de continuar tras el ideal de la definición universal de especie es si es posible llegar a esta meta. Desde un punto de vista filosófico los biólogos tratan de alcanzar una definición que sería en nuestro caso: "una proposición clara, exacta y concisa que expone los caracteres genéricos y diferenciales de algo y da a conocer su naturaleza" (Cabanellas 1988). Sin embargo, pocos saben lo que es una proposición en términos de lógica, así una proposición es un enunciado que se puede determinar si es falso o verdadero (Góngora 1983). En consecuencia nuestra definición de especie debe incluir una claridad tal que podamos determinar si lo dicho es falso o verdadero, cosa que no sucede ya que todas caen en el ámbito especulativo, al no existir una lógica clara de la separación y quedar por fuera algunas dimensiones necesarias para poder hablar de una definición.

Quizás sería importante que exploremos que es una definición ya que solemos utilizar términos muy a la ligera y muchas veces no cumplimos los requisitos de esos términos. Analizando el trabajo de los filósofos dedicados a la lógica obtenemos lo siguiente: toda definición establece una relación de equivalencia entonces si $\mathrm{x} \equiv$ def. $\mathrm{y}$, entonces dondequiera que haya $\mathrm{x}$ podremos poner y (Camacho 1983), entonces podríamos construir la siguiente oración: "Las plantas se hallan divididas en unidades naturales que son especies" y entonces si por ejemplo aceptamos la definición de especie de Mayr, podremos decir lo siguiente sin temor a error "Las plantas se hallan divididas en unidades naturales que son grupos de poblaciones naturales que se entrecruzan $y$ que están reproductivamente aislados de otros grupos" y esto es falso, ya que entonces los robles y otras especies no serían especies. Si aplicamos el principio del contraejemplo (utilizado para la demostración en matemática) (Britton et al. 1969), así la conjetura (=proposición que no ha sido demostrada) (Britton et al. 1969) planteada por Mayr (1969) como definición de especie no lo es. Este ejercicio podemos hacerlo con cada una de las conjeturas 
que han sido planteadas como definiciones de especie, obteniendo el mismo resultado, pero dejaremos esta práctica a los lectores.

Entonces ¿qué es una definición?, posiblemente algo más complejo de lo que hasta el momento se ha hecho. Según Camacho (1983) las reglas para formular definiciones son: 1debe indicar atributos esenciales de lo definido, entendiendo por esencial aquello que es propio de lo definido, de tal modo que lo distingue de todas las demás cosas (biológicamente ha sido imposible hallar esta esencia de la especie, que por demás convierte a la definición en tipológica), 2- la definición no debe ser circular, es decir que lo definido no debe estar incluido en la definición, 3- no debe ser demasiado ancha ni demasiado estrecha, esto es consecuencia de (1), ya que si se han utilizado los atributos esenciales la definición no será ni muy ancha ni muy estrecha, obviamente Mayr no concibió una buena definición, ya que es muy estrecha, en consecuencia lo que este autor consideró esencial no lo es, 4- no debe utilizarse lenguaje ambiguo, oscuro o figurado y 5- no debe ser negativa, siempre y cuando pueda se positiva.

De todo esto se deriva que las definiciones de especie existentes no lo son, y la forma real de construirla sería por intensión, sin embargo la amplitud de la diversidad de la vida no permite este tipo de determinación y en consecuencia no es lógico continuar buscando una definición de especie.

En consecuencia si se analiza lo que debe ser una definición implica el poder encontrar una suerte de fórmula que nos permita determinar que cosa son los caracteres de una "especie" sin importar su naturaleza biológica, es decir que podemos determinar cuál es esa esencia que hace que A sea una especie y B otra y no una subespecie (regla 1)

Atendiendo a este razonamiento al lograr la verdadera definición de especie habríamos encontrado ese "grupo" de características universales para poder ubicar sin temor a error a cualquier ser vivo en su correspondiente categoría específica, en cualquier punto del univer- so y en cualquier tiempo, en otras palabras habríamos alcanzado uno de los ideales de los filósofos griegos: la esencia de la diferenciación de la vida, o lo que se ha llamado tipologismo.

En este punto se contradice el pensamiento biológico como tal. Los que se han dedicado al estudio de la evolución y en particular a los procesos de selección habitualmente se oponen a las descripciones tipológicas que desestiman caracteres que a juicio del taxónomo no son relevantes, pero podrían serlo a nivel biológico. En consecuencia se aboga por la no práctica del tipologismo, por lo que se exige describir en función de ámbitos de variación de los caracteres que permitan observar la variabilidad interna del grupo determinado como especie. La aceptación de la variabilidad, indispensable dentro de la concepción biológica de las poblaciones, se opone al esencialismo tipológico de la siguiente forma:

Caso 1: Una especie cualquiera, llamada aquí $\mathrm{X}$ es definida como variable en razón del número de antenómeros presentes. Se determina inicialmente que el número de antenómeros varía entre 7 y 8 . La posterior revisión del material que condujo a la anterior aseveración nos lleva luego a la conclusión de que la especie $\mathrm{X}$ cuenta siempre son 7 antenómeros, y que el grupo de individuos con 8 antenómeros es una nueva especie en realidad.

Si el primer taxónomo hubiera actuado siguiendo los preceptos de la doctrina escencialista, debió pensar que ambas condiciones del número de antenómeros correspondían a escencias diferentes y en consecuencia a diferentes especies. En consecuencia la determinación de los ámbitos de variación poblacional obedece a un criterio totalmente subjetivo, y entonces la reducción o ampliación de ese ámbito obedece a un criterio individual.

Entonces si se ha luchado para erradicar el tipologismo por ser una medida equivocada de la población, como pretendemos encasillar en unos cuantos caracteres la esencia de todas las especies que existen, que existieron y que existirán, donde quiera que haya vida. Es obvio que si la consideración de los caracteres esenciales 
es subjetiva y varía según los conceptos de prioridad de cada individuo, entonces la esencia tipológica varía con cada cual que analiza el problema, en consecuencia las "definiciones de especie" serán tantas como especialistas del concepto existan y por tanto es imposible llegar a tal definición universal de especie.

Utilidad biológica del proceso lógico planteado: Si no es posible dentro de los mecanismos conocidos por nuestra forma de acceso al conocimiento llegar a una verdadera definición de especie. Entonces es posible que ese camino esté equivocado, analizado el problema de la siguiente forma: a) aunque los mecanismos de especiación son siempre los mismos para cualquier ser vivo existen variables que alteran el resultado del mecanismo, b) la fijación de un cambio que pueda determinar una novedad evolutiva es de diferente magnitud en cada grupo de organismos, c) el tiempo de fijación efectiva es diferente en cada población, d) la magnitud de un cambio tiene un valor relativo diferente para cada población, pudiendo producir segregación específica en una población A y no en una población B, bajo diferentes condiciones biológicas.

Este entorno altera por completo los resultados de los procesos de especiación, entonces el mecanismo es el mismo, pero los resultados totalmente impredecibles, porque el mecanismo será alterado por n-dimensiones biológicas propias de cada población, donde el éxito reproductivo de un solo organismo puede no tener impacto, o ser totalmente decisivo en la cladogénesis. En consecuencia la predicción de la especiación es semejante a la predicción meteorológica, en realidad son sistemas caóticos y por tanto no predecibles y con la posibilidad de que cualquier detalle por ínfimo que parezca pueda ser determinante en un caso y totalmente inocuo en otro, en estas condiciones es imposible hallar una definición que abarque toda posibilidad. Ejemplos de esto son los siguientes:

Caso 2: Existiendo una especie A, en la cual las barreras para la posible fertilización cruzada con individuos de cualquier otra especie manifiestan una efectividad de $100 \%$. Esto significa que no existirá nunca un híbrido de A contra cualquier otra especie.

Caso 3: Existiendo $n$ especies demostradamente emparentadas distribuidas a lo largo del planeta, se evidencia que si se mantienen cercanas son capaces de hibridar y obtener una descendencia fértil entre ellas, a pesar de mantener cada una de ellas poblaciones totalmente cohesivas (Brooks y Wiley 1986, Templeton 1983, Demetrius 2000).

Utilizando la lógica de Mayr (1969) el caso 2 evidencia una especie y el caso 3 igualmente evidencia una especie. Sin embargo sabemos que en el segundo caso son igualmente especies con otra realidad biológica que no desembocó en barreras de fertilización o canalización embrionaria. En consecuencia la lógica de Mayr para la definición de especie será útil en algunos casos y completamente inútil en otros, por lo tanto por contradicción no es una definición al no abarcar todo posible caso en cualquier circunstancia. Esto se repite con cada una de las definiciones intentadas, en consecuencia no existe tal definición.

Entonces quizás sería mejor no intentar una definición, sino una misma lógica de segregación, además como se apuntó en la introducción hay problemas de consideración en las descripciones al tomar en cuenta el tipo de epifenotipo que sirvió de patrón para la decripción. Pero esto va aún más allá, actualmente sabemos que un análisis filogenético de estados inmaduros arroja resultados diferentes en parentescos a los que arroja un análisis de adultos (Papavero et al. 1993)

Esto no implica que las relaciones filogenéticas cambien con la ontogenia sino, que las presiones bióticas y abióticas sobre los diferentes caracteres si se ven alteradas por el epifenotipo escogido para el análisis, ya que como es obvio no es igual la presión sobre una larva fitófaga que sobre un adulto nectarívoro a nivel de forrageo, así un tóxico almacenado en las hojas afectará a las larvas pero no necesariamente a los adultos y viceversa.

Siendo esto así, es aún más complejo el problema ya que la presión de selección puede estar actuando donde no somos capaces de 
suponerla y un cambio a nivel de un determinado epifenotipo puede llevar a una cladogénesis sin afectar en nada las barreras de segregación, y entonces cualquier definición de especie en función de estas barreras es inútil como definición.

Dentro de esto si todos los taxónomos adoptamos una única lógica de separación de los taxa desde especie hasta reino en la que siempre se cumpla que: a) se está ante un nuevo taxon si y solo si este cuenta con una o más de una apomorfía o cuenta con una combinación novedosa de caracteres que lo hace distinto a cualquier otra descripción absoluta de un taxon previamente descrito (condición vi de la postulación matemática precedente), b) las diagnosis de los diferentes taxa sin importar su nivel jerárquico deben ser absolutas en cuanto a la necesidad de la existencia de al menos un caracter o combinación de caracteres que forzosamente esté presente en todos y cada uno de los constituyentes del taxon, este caracter o combinación de caracteres será el absoluto de la diagnosis y el único crítico en última instancia, c) los taxa se segregan ya sea porque algún o algunos de sus miembros cuentan con nuevas apomorfías que como eventos evolutivos justifican la segregación o porque algún o algunos miembros carecen de caracteres apomórficos del taxon y al ser taxa con estados plesiomórficos no pueden agruparse con los que si los tienen, d) todo caracter de un taxon que sea apomórfico debe considerarse absoluto del taxon (Hennig 1968, Retana-Salazar 1998) y por lo tanto crítico en la segregación y e) toda relación de subordinación de taxa debe estar forzosamente condicionada por una estricta relación de c-inclusión, es decir que estén ligados por la inclusión forzada de los caracteres.

Si todo taxónomo sigue una lógica de separación fundamentada en la lógica matemática no garantiza la ausencia de errores, pero si 1) una mayor facilidad en las revisiones taxonómicas, 2) un mayor orden en las descripciones, 3) una más elaborada técnica de discernimiento crítico de caracteres absolutos y 4) la erradicación de taxa con límites indefinidos por la ausencia de la definicón crítica absoluta de los mismos.
Hacia una nueva sintaxis: por último, se hace indispensable la utilización de una nueva sintaxis que permita generar frases en el lenguaje de la evolución con su consecuente gramática, como lo proponen Brooks y Wiley (1986) y Papentin (1980) quien creó un lenguaje simbólico que permite intentar esta gramática generativa de la evolución, dentro de lo cual tenemos que si representamos con w a una palabra cualquiera del lenguaje evolutivo que sería lo mismo que un taxon, esta palabra sería un elemento de L, donde L sería el lenguaje en su totalidad (=evolución), donde el lenguaje sería un subconjunto de un alfabeto terminal $\left(\mathrm{A}_{\mathrm{T}}\right)$, este alfabeto en el cual no existe el conjunto vacío, está constituido por los caracteres de cada taxon.

Sabiendo que toda teoría biológica de la sistemática debe partir de la misma base empírica que es la existencia de un conjunto de caracteres que identifican a un grupo particular de especies, y así el método será desarrollado considerando una estructura sintáctica definida en función de a y s (siendo a el ancestro o punto de enraizamiento) y s (las reglas que definen la construcción idiomática) (Brooks y Wiley 1986).

Función a: Aunque Brooks y Wiley (1986) porponen el uso del grupo externo (=outgroup) como un estimador del posible origen de los grupos, o una forma de establecer la ancestría, hay casos en los que esto puede llevar a errores graves.

Por ejemplo, hace 10 años pocos hubieran dudado en utilizar como grupo externo de Ichneumonidae (Insecta: Hymenoptera) a la familia que suponemos más cercana Braconidae (Insecta: Hymenoptera), gracias a los estudios de Coronado (en preparación) hoy sabemos que Braconidae es un grupo de Ichneumonidae y en consecuenia no puede actuar como un grupo externo.

Tomando en cuenta que por (v) de la postulación matemática presentada, la unidad evolutiva denominada como especie está definida en función de 3 magnitudes que actúan como dimensiones biológicas, podemos decir en función de la teoría matemática que fundamenta 
los cálculos de vectores que el conjunto de unidades evolutivas descritas por estas tres dimensiones conformarán una matriz en la cual el vector solución será equivalente al vector ancestro. Para esto se puede aplicar cualquier método de resolución de matrices no cuadradas para vectores independientes.

Este método se describirá en detalle en futuras publicaciones sobre el tema de la aplicación práctica del álgebra lineal en su resolución de matrices a los conceptos sistemáticos, sin perder de vista los requerimientos biológicos de estos conceptos.

Función s: la lógica determinada en este aspecto por Brooks y Wiley (1986) es pertinente para nuestro caso, por lo que la respetaremos en su totalidad.

\section{RESUMEN}

En este artículo discutimos la utilidad del concepto de especie como una definición real, particularmente el concepto de Mayr. Proponemos un método de separación lógica de los taxa basada en planteamientos de la lógica matemática y la aplicación de la teoría de los conjuntos a los conceptos en sistemática. Intentamos de esta manera proporcionar una metodología objetiva para la interpretación de los grupos naturales en biología incluyendo la especie como un grupo básico en evolución. Introducimos el concepto de ancestro hipotético como una posibilidad matemática derivada del uso del cálculo de matrices no cuadradas.

\section{REFERENCIAS}

Brook, D.R. \& E.O. Wiley. 1986. Evolution as Entropy: Toward a Unified Theory of Biology, Chicago University. $335 \mathrm{p}$.
Britton, J.R., R Ben Kriegh \& L.W.,Rutland. 1969. Matemáticas Universitarias, Tomo 1, C.E.C.S.A., México. $747 \mathrm{p}$.

Cabanellas de Torres, G. 1988. Diccionario Jurídico Elemental. Editorial Heliasta, Argentina.

Camacho, L. 1983. Introducción a la lógica. Tecnológica de Costa Rica, Costa Rica. 244 p.

Demetrius, L. 2000. Thermodynamics and Evolution. J. theor. Biol. 206: 1-16.

Góngora, E. 1983. Introducción al pensamiento lógico matemático. EUNED, San José, Costa Rica. 201 p.

Hennig, W. 1968. Elementos de una sistemática filogenética. Editorial Universitaria de Buenos Aires, Buenos Aires, Argentina.

Mayr, E. 1969. Principles of Systematic Zoology. McGraw-Hill Inc. New York. 26 p.

Papavero, N., J. Llorente-Bousquets \& J. Minoro-Abe. 1993. Principia taxonomica: Una introducción a los fundamentos lógicos, filosóficos y metodológicos de las escuelas de taxonomía biológica. Vol I. Conceptos básicos de taxonomía: una formalización. Cap. 2. El semaforonte, base de la sistematica biológica. pp. 29-33.

Papentin, F. 1980. On order and complexity. 1. General considerations. J. theor. Biol. 87: 421-456.

Retana-Salazar, A.P. 1998. Una visión filogenética del género Frankliniella (Thysanoptera: Thripidae). Rev. Biol. Trop. 46: 397-406.

Templeton, A.R. 1983. Phylogenetic inference from restriction endonuclease cleavage site maps with particular reference to the humans and apes. Evolution 37: 221-244.

Torrey, T.W. 1993. Organisms in time. Quart. Rev. Biol. 14: $275-288$. 


\section{APENDICE 1}

\section{Lista de símbolos y definiciones utilizados en este trabajo}

\author{
Símbolos \\ $=\quad$ es igual a... \\ $\neq \quad$ no es igual a... \\ es equivalente a... \\ $>\quad$ mayor que... \\ $\geq$ mayor que o igual a... \\ $\varnothing \quad$ conjunto vacío \\ $\cap \quad$ intersección de conjuntos \\ A-B diferencia de conjuntos \\ $\langle\ldots\rangle$ conjunto \\ $\Rightarrow$ implica que... \\ $\Leftrightarrow \quad$ si y solo si... \\ $\forall \quad$ para todo... \\ $\exists \quad$ existe un... \\ $\wedge \quad \mathrm{y} \ldots$

\section{Definiciones}

Cota inferior $=$ sea $S$ un conjunto no vacío suponemos que existe $\mathrm{B}$ tal que $\mathrm{X} \geq \mathrm{B}$ para todo $\mathrm{x}$ de $\mathrm{S}$. Entonces se dice que $\mathrm{S}$ está acotado inferiormente por B y B es una cota inferior de $\mathrm{S}$ $\mathrm{y}$ todo elemento menor que $\mathrm{B}$ será igualmente una cota inferior de $\mathrm{S}$. Si además B pertenece a $\mathrm{S}$, entonces $\mathrm{B}$ se denomina elemento ínfimo de $\mathrm{S}$, a lo sumo puede existir un elemento B que sea elemento ínfimo de $\mathrm{S}(\mathrm{B}=$ inf. $\mathrm{S})$.

Conjunto reticulado $=$ se dice del conjun to donde por relación de orden cada elemento contiene los carateres del elemento anterior y al menos uno más estableciendo una relación de inclusión o "parentesco".

Cadena $($ linaje $)=$ cuando el conjunto reticulado está constituído por $n$ elementos $(n \geq 1)$ donde todo elemento superior a otro contiene todos los mismos caracteres que los inmediatos anteriores y al menos uno más (entendiéndose como caracter algo novedoso o una combinación de carcateres novedosa).

Conjunto de cadenas $($ genos $)=$ cuando al menos un caracter de un elemento $\mathrm{x}$ de una cadena A también es compartido por los elementos de otra cadena $\mathrm{B}$, entonces $\mathrm{x}$ pertenece a las dos cadenas A y B que comparten entre sí caracteres pero no son la misma ya que $A \neq B$, este agrupamiento de cadenas en función del "parentesco" es conocido como genos.

Inf-reticulado $=$ se dice del conjunto reticulado que presenta un elemento ínfimo, se dice entonces que para cualquier sistema vivo de organismos el inf-elemento contiene los caracteres elementales o básicos de la cadena que de este se origine estableciendo la relación ancestro, y entonces el inf-elemento de un reticulado contendrá los caracteres básicos del reticulado y será equivalente an ancestro del conjunto reticulado, que será un genos en función de la unión de los diferentes linajes (grupo monofilético). 